\title{
A NOTE ON THE MIXTURE REPRESENTATION OF THE CONDITIONAL RESIDUAL LIFETIME OF A COHERENT SYSTEM
}

\author{
XIUYING FENG * AND \\ SHUHONG ZHANG, ${ }^{* * *}$ Lanzhou University \\ XIAOHU LI, ${ }^{* * *}$ Xiamen University
}

\begin{abstract}
This paper builds a mixture representation of the reliability function of the conditional residual lifetime of a coherent system in terms of the reliability functions of conditional residual lifetimes of order statistics. Some stochastic ordering properties for the conditional residual lifetime of a coherent system with independent and identically distributed components are obtained, based on the stochastically ordered coefficient vectors.

Keywords: Coherent system; order statistics; residual lifetime; signature; stochastic order 2010 Mathematics Subject Classification: Primary 62E15

Secondary $60 \mathrm{~K} 10$
\end{abstract}

\section{Introduction}

A system of components is said to be coherent if each of the components is relevant (this means that the performance of a component does effect the performance of the system) and its structure function is monotone in each argument. It is very important to study the stochastic behavior and the ageing property of a coherent system in reliability engineering.

Since the coherent structure may be very complex, many researchers have compared the performance of competing systems by means of the mixture representation of its reliability function. Consider a coherent system consisting of $n$ components whose lifetimes $X_{1}, X_{2}, \ldots, X_{n}$ are independent and identically distributed (i.i.d.) with a common distribution function $F$, let $T(X)=T\left(X_{1}, \ldots, X_{n}\right)$ denote the lifetime of the system. Samaniego [16] (see also [9]) proved that the reliability function of the system $T(X)$ can be expressed as a mixture of the survival functions of order statistics with respect to its signature when $F$ is continuous, that is,

$$
\mathbb{P}(T(\boldsymbol{X})>t)=\sum_{i=1}^{n} p_{i} \mathbb{P}\left(X_{i: n}>t\right),
$$

where $X_{i: n}$ is the $i$ th smallest order statistic among $X_{1}, X_{2}, \ldots, X_{n}$, and $\boldsymbol{p}=\left(p_{1}, \ldots, p_{n}\right)$ with $p_{i}=\mathbb{P}\left(T(\boldsymbol{X})=X_{i: n}\right)$ is called the signature of the system. For more details on this topic, we refer the reader to [4], [5], [10], [11], [13], [14], [15], [17], [20], and [22].

Many authors have studied various types of residual lifetime and inactivity time of coherent systems in the past decade. See, for instance, [3], [7], [18], [20], and [22]. Recently, Navarro et al. [13] represented the reliability functions of the residual lifetime $[T(\boldsymbol{X})-t \mid T(\boldsymbol{X})>t]$ and

Received 13 September 2012; revision received 14 October 2012.

* Postal address: School of Mathematics and Statistics, Lanzhou University, Lanzhou 730000, China.

** Email address: shuhongzhang@1zu.edu.cn

*** Postal address: School of Mathematics Sciences, Xiamen University, Xiamen 361005, China. 
conditional residual lifetime $\left[T(\boldsymbol{X})-t \mid T(\boldsymbol{X})>t, X_{k: n} \leq t\right]$ of coherent systems in terms of the mixture of reliability functions of residual lifetimes of order statistics, i.e.

$$
\mathbb{P}(T(\boldsymbol{X})-t>x \mid T(\boldsymbol{X})>t)=\sum_{i=1}^{n} s_{i}(t) \mathbb{P}\left(X_{i: n}-t>x \mid X_{i: n}>t\right),
$$

where $s_{i}(t)=\mathbb{P}\left(T(\boldsymbol{X})=X_{i: n} \mid T(\boldsymbol{X})>t\right)$ and $\sum_{i=1}^{n} s_{i}(t)=1$, and

$$
\mathbb{P}\left(T(\boldsymbol{X})-t>x \mid T(\boldsymbol{X})>t, X_{k: n} \leq t\right)=\sum_{j=1}^{n} s_{j}(t, k) \mathbb{P}\left(X_{j: n}-t>x \mid X_{j: n}>t\right),
$$

where $s_{1}(t, k), \ldots, s_{n}(t, k)$, which implicitly depend on $F$, are real numbers such that $\sum_{j=1}^{n} s_{j}(t, k)=1$. Suppose that $T_{i}(\boldsymbol{X})$ is the lifetime of a coherent system with i.i.d. components having lifetimes $X_{1}, X_{2}, \ldots, X_{n}$ and corresponding mixing coefficients vector $s_{i}(t), i=1,2$. Furthermore, Navarro et al. [13] also established that $s_{1}(t) \leq_{\mathrm{st}}\left(\leq_{\mathrm{hr}}, \leq_{\mathrm{lr}}\right) \boldsymbol{s}_{2}(t)$ implies that

$$
\left[T_{1}(X)-t \mid T_{1}(X)>t\right] \leq_{\mathrm{st}}\left(\leq_{\mathrm{hr}}, \leq_{\mathrm{lr}}\right)\left[T_{2}(\boldsymbol{X})-t \mid T_{2}(\boldsymbol{X})>t\right] .
$$

However, just as illustrated by Example 3.2 of Navarro et al. [13], not all coefficients of $\boldsymbol{s}(t, k)=\left(s_{1}(t, k), \ldots, s_{n}(t, k)\right)$ in (1.2) are necessarily nonnegative and, hence, it does not denote the distribution of an arithmetic random variable anymore. Consequently, (1.2) cannot be used to obtain stochastic comparison results except when all mixing coefficients are nonnegative.

In this paper we further investigate the mixture representation of the conditional residual lifetime of a coherent system of type $\left[T(\boldsymbol{X})-t \mid T(\boldsymbol{X})>t, X_{k: n} \leq t\right]$. The rest of this paper is organized as follows. In Section 2 we introduce some stochastic orders to be used throughout this paper and build several useful lemmas to be utilized in proving our main conclusions. In Section 3, we present a new mixture representation of the conditional residual lifetime of a coherent system in terms of conditional residual lifetimes of order statistics, and then we build stochastic comparisons on the conditional residual lifetimes of coherent systems consisting of the same group of independent components with identically distributed lifetimes. Finally, we obtain stochastic order properties of conditional residual lifetimes of coherent systems consisting of components with their identically distributed lifetimes being stochastically ordered.

Throughout the paper, we use the term increasing and decreasing in place of nondecreasing and nonincreasing, respectively, all components of a concerned system are independent and identical, all random variables under consideration are absolutely continuous and have 0 as the common left endpoint of their supports, and expectations are finite as they appear.

\section{Preliminaries and lemmas}

For two random variables $X$ and $Y$ with respective distribution functions $F$ and $G$, denote their respective probability density functions by $f$ and $g$, and let $\bar{F}=1-F$ and $\bar{G}=1-G$ be the corresponding reliability functions.

Definition 2.1. The random variable $X$ is said to be smaller than $Y$ in the

(i) usual stochastic order (denoted by $X \leq_{\text {st }} Y$ ) if $\bar{G}(x) \geq \bar{F}(x)$ for all $x$;

(ii) hazard rate order (denoted by $X \leq \mathrm{hr} Y$ ) if $\bar{G}(x) / \bar{F}(x)$ is increasing in $x$;

(iii) likelihood ratio order (denoted by $X \leq \operatorname{lr} Y$ ) if $g(x) / f(x)$ is increasing in $x$. 
An arithmetic random variable only takes some positive integer as its possible value. This note concerns the stochastic comparison of arithmetic random variables with possible values in $\{1, \ldots, n\}$, where $n$ usually denotes the number of components in a coherent system. For briefness, we directly define the following orders on their probability vector instead.

Definition 2.2. A probability vector $\boldsymbol{p}=\left(p_{1}, \ldots, p_{n}\right)$ is said to be smaller than $\boldsymbol{q}=$ $\left(q_{1}, \ldots, q_{n}\right)$ in the

(i) usual stochastic order (denoted by $\boldsymbol{p} \leq_{\mathrm{st}} \boldsymbol{q}$ ) if $\sum_{j=i}^{n} q_{j} \geq \sum_{j=i}^{n} p_{j}$ for all $i=1,2, \ldots, n$;

(ii) hazard rate order (denoted by $\boldsymbol{p} \leq_{\mathrm{hr}} \boldsymbol{q}$ ) if $\sum_{j=i}^{n} q_{j} / \sum_{j=i}^{n} p_{j}$ is increasing in $i$;

(iii) likelihood ratio order (denoted by $\boldsymbol{p} \leq_{\mathrm{lr}} \boldsymbol{q}$ ) if $q_{i} / p_{i}$ is increasing in $i$, when $p_{i}, q_{i}>0$.

The following several lemmas will be useful in establishing our main results.

Lemma 2.1. ([12].) Assume that $\Theta$ is a subset of the real line $\mathbb{R}$, and that $U$ is a nonnegative random variable whose distribution belongs to the family $\mathcal{H}=\{H(\cdot \mid \theta): \theta \in \Theta\}$, which satisfies, for $\theta_{1}, \theta_{2} \in \Theta$,

$$
H\left(\cdot \mid \theta_{1}\right) \leq_{\mathrm{st}}\left(\geq_{\mathrm{st}}\right) H\left(\cdot \mid \theta_{2}\right) \quad \text { whenever } \theta_{1}<\theta_{2} .
$$

Let $\psi(u, \theta)$ be a real-valued function defined on $\mathbb{R} \times \Theta$, which is measurable in $u$ for each $\theta$ such that $\mathbb{E}_{\theta}[\psi(U, \theta)]$ exists. Then $\mathbb{E}_{\theta}[\psi(U, \theta)]$ is

(i) increasing in $\theta$ if $\psi(u, \theta)$ is increasing in $\theta$ and increasing (decreasing) in $u$; and

(ii) decreasing in $\theta$ if $\psi(u, \theta)$ is decreasing in $\theta$ and decreasing (increasing) in $u$.

Lemma 2.2. ([6].) Let $A, B$, and $C$ be subsets of the real line. Let $L(x, z)$ be $\mathrm{SR}_{2}$ (sign regular of order 2) for $x \in A$ and $z \in B$, and let $M(z, y)$ be $\mathrm{SR}_{2}$ for $z \in B$ and $y \in C$. Then, for any $\sigma$-finite measures $\mu(z)$,

$$
K(x, y)=\int_{B} L(x, z) M(z, y) \mathrm{d} \mu(z)
$$

is also $\mathrm{SR}_{2}$ for $x \in A$ and $y \in C$ and $\varepsilon_{i}(K)=\varepsilon_{i}(L) \varepsilon_{i}(M)$ for $i=1,2$, where $\varepsilon_{i}(K)=\varepsilon_{i}$ denotes the constant sign of the $i$-order determinant.

Lemma 2.3. Let $\phi_{1}(t)=F(t) / \bar{F}(t)$ and $\phi_{2}(t)=G(t) / \bar{G}(t)$. If $X \leq_{\mathrm{st}} Y$ then

$$
\lambda_{t}(u)=\frac{\sum_{l=k}^{j-1}\left(\begin{array}{c}
n \\
l
\end{array}\right)\left(\begin{array}{c}
n-l \\
j-l
\end{array}\right)(j-l) \phi_{2}^{l}(t) u^{j-l-1}}{\sum_{l=k}^{j-1}\left(\begin{array}{c}
n \\
l
\end{array}\right)\left(\begin{array}{c}
n-l \\
j-l
\end{array}\right)(j-l) \phi_{1}^{l}(t) u^{j-l-1}}
$$

is increasing in $u \in \mathbb{R}_{+}$for each $t>0$ and any integers $j$ and $k$ such that $1 \leq k<j$.

Proof. For $u \in \mathbb{R}_{+}$and $t>0$, define

$$
\Phi_{i}(t, u)=\sum_{l=k}^{j-1}\left(\begin{array}{l}
n \\
l
\end{array}\right)\left(\begin{array}{c}
n-l \\
j-l
\end{array}\right)(j-l) \phi_{i}^{l}(t) u^{j-l-1}, \quad i=1,2 .
$$

Then $\lambda_{t}(u)$ can be rewritten as

$$
\lambda_{t}(u)=\frac{\Phi_{2}(t, u)}{\Phi_{1}(t, u)}, \quad u \in \mathbb{R}_{+} \text {and } t>0 .
$$


If $X \leq_{\text {st }} Y$ then $\phi_{2}(t) \leq \phi_{1}(t)$ for all $t>0$, and, hence, $\phi_{i}^{l}(t)$ is $\mathrm{RR}_{2}$ (reverse regular of order $2)$ in $(i, l) \in\{1,2\} \times \overline{\mathbb{N}}$ for each fixed $t>0$. Moreover, it is easy to see that $u^{j-l-1}$ is $\mathrm{RR}_{2}$ in $(l, u) \in \mathbb{N} \times \mathbb{R}_{+}$for each fixed $j \in \mathbb{N}$. Therefore, by Lemma 2.2, $\Phi_{i}(t, u)$ is $\operatorname{TP}_{2}$ (totally positive of order 2$)$ in $(i, u) \in\{1,2\} \times \mathbb{R}_{+}$for each fixed $t>0$. That is, $\lambda_{t}(u)$ is increasing in $u \in \mathbb{R}_{+}$for fixed $t>0$.

\section{Main results}

Let $T(X)=T\left(X_{1}, \ldots, X_{n}\right)$ be the lifetime of a coherent system with i.i.d. component lifetimes $X_{1}, X_{2}, \ldots, X_{n}$ from a continuous distribution function $F$. Let $\bar{F}=1-F$ be the common reliability functions, and let $X_{1: n}, X_{2: n}, \ldots, X_{n: n}$ be the corresponding order statistics. In this section we study the residual lifetime of a coherent system when the system is working and at least $k$ of the components have failed at time $t$, i.e. the conditional random variable $\left[T(\boldsymbol{X})-t \mid T(\boldsymbol{X})>t, X_{k: n} \leq t\right]$. First, a mixture representation for the reliability function of the conditional residual lifetime of a coherent system is presented.

Theorem 3.1. Suppose that a coherent system has lifetime $T(X)$ and signature $\boldsymbol{p}$, and that $\mathbb{P}\left(T(X)>t, X_{k: n} \leq t\right)>0$ for some $k \in\{1, \ldots, n-1\}$. Then, for all $x \geq 0$,

$$
\begin{aligned}
& \mathbb{P}\left(T(\boldsymbol{X})-t>x \mid T(\boldsymbol{X})>t, X_{k: n} \leq t\right) \\
& \quad=\sum_{i=k+1}^{n} p_{i}(t, k) \mathbb{P}\left(X_{i: n}-t>x \mid X_{i: n}>t, X_{k: n} \leq t\right),
\end{aligned}
$$

where $\boldsymbol{p}(t, k)=\left(0, \ldots, 0, p_{k+1}(t, k), \ldots, p_{n}(t, k)\right)$ with

$$
\begin{aligned}
p_{j}(t, k) & =\mathbb{P}\left(T(\boldsymbol{X})=X_{j: n} \mid T(\boldsymbol{X})>t, X_{k: n} \leq t\right) \\
& =\frac{p_{j} \mathbb{P}\left(X_{j: n}>t, X_{k: n} \leq t\right)}{\sum_{i=k+1}^{n} p_{i} \mathbb{P}\left(X_{i: n}>t, X_{k: n} \leq t\right)}
\end{aligned}
$$

such that $\sum_{j=k+1}^{n} p_{j}(t, k)=1$.

Proof. By the total probability law,

$$
\begin{gathered}
\mathbb{P}\left(T(\boldsymbol{X})-t>x \mid T(\boldsymbol{X})>t, X_{k: n} \leq t\right) \\
=\sum_{j=k+1}^{n} \mathbb{P}\left(T(\boldsymbol{X})-t>x, T(\boldsymbol{X})=X_{j: n} \mid T(\boldsymbol{X})>t, X_{k: n} \leq t\right) \\
=\sum_{j=k+1}^{n} \mathbb{P}\left(T(\boldsymbol{X})-t>x \mid T(\boldsymbol{X})>t, T(\boldsymbol{X})=X_{j: n}, X_{k: n} \leq t\right) \\
\quad \times \mathbb{P}\left(T(\boldsymbol{X})=X_{j: n} \mid T(\boldsymbol{X})>t, X_{k: n} \leq t\right) \\
=\sum_{j=k+1}^{n} \mathbb{P}\left(X_{j: n}-t>x \mid X_{j: n}>t, T(\boldsymbol{X})=X_{j: n}, X_{k: n} \leq t\right) \\
\quad \times \mathbb{P}\left(T(\boldsymbol{X})=X_{j: n} \mid T(\boldsymbol{X})>t, X_{k: n} \leq t\right) .
\end{gathered}
$$


By the independence of the order statistics with their ranks (see, e.g. [9]), we have

$$
\begin{aligned}
& \mathbb{P}\left(T(\boldsymbol{X})-t>x \mid T(\boldsymbol{X})>t, X_{k: n} \leq t\right) \\
& \quad=\sum_{j=k+1}^{n} \mathbb{P}\left(X_{j: n}-t>x \mid X_{j: n}>t, X_{k: n} \leq t\right) \mathbb{P}\left(T(\boldsymbol{X})=X_{j: n} \mid T(\boldsymbol{X})>t, X_{k: n} \leq t\right) .
\end{aligned}
$$

Define, for $j=k+1, \ldots, n$,

$$
\begin{aligned}
p_{j}(t, k) & =\mathbb{P}\left(T(\boldsymbol{X})=X_{j: n} \mid T(\boldsymbol{X})>t, X_{k: n} \leq t\right) \\
& =\frac{\mathbb{P}\left(T(\boldsymbol{X})=X_{j: n}, T(\boldsymbol{X})>t, X_{k: n} \leq t\right)}{\mathbb{P}\left(T(\boldsymbol{X})>t, X_{k: n} \leq t\right)} \\
& =\frac{\mathbb{P}\left(T(\boldsymbol{X})=X_{j: n}\right) \mathbb{P}\left(T(\boldsymbol{X})>t, X_{k: n} \leq t \mid T(\boldsymbol{X})=X_{j: n}\right)}{\sum_{i=k+1}^{n} \mathbb{P}\left(T(\boldsymbol{X})>t, T(\boldsymbol{X})=X_{i: n}, X_{k: n} \leq t\right)} \\
& =\frac{\mathbb{P}\left(T(\boldsymbol{X})=X_{j: n}\right) \mathbb{P}\left(X_{j: n}>t, X_{k: n} \leq t\right)}{\sum_{i=k+1}^{n} \mathbb{P}\left(T(\boldsymbol{X})=X_{i: n}\right) \mathbb{P}\left(T(\boldsymbol{X})>t, X_{k: n} \leq t \mid T(\boldsymbol{X})=X_{i: n}\right)} \\
& =\frac{\mathbb{P}\left(T(\boldsymbol{X})=X_{j: n}\right) \mathbb{P}\left(X_{j: n}>t, X_{k: n} \leq t\right)}{\sum_{i=k+1}^{n} \mathbb{P}\left(T(\boldsymbol{X})=X_{i: n}\right) \mathbb{P}\left(X_{i: n}>t, X_{k: n} \leq t\right)} \\
& =\frac{p_{j} \mathbb{P}\left(X_{j: n}>t, X_{k: n} \leq t\right)}{\sum_{i=k+1}^{n} p_{i} \mathbb{P}\left(X_{i: n}>t, X_{k: n} \leq t\right)} .
\end{aligned}
$$

Then

$$
\sum_{j=k+1}^{n} p_{j}(t, k)=\frac{\sum_{j=k+1}^{n} p_{j} \mathbb{P}\left(X_{j: n}>t, X_{k: n} \leq t\right)}{\sum_{i=k+1}^{n} p_{i} \mathbb{P}\left(X_{i: n}>t, X_{k: n} \leq t\right)}=1 .
$$

This completes the proof.

Theorem 3.1 represents the conditional residual lifetime $\left[T(X)-t \mid T(X)>t, X_{k: n} \leq t\right]$ of a coherent system at time $t$ as a mixture of the conditional residual lifetime $\left[X_{j: n}-\right.$ $\left.t \mid X_{j: n}>t, X_{k: n} \leq t\right]$ of order statistics (that is, the lifetimes of $k$-out-of- $n$ systems) through the coefficients $p_{j}(t, k)$. Note that the vector of coefficients $\boldsymbol{p}(t, k)$ depends only on the structure of the system and the distribution function of the components; see some examples in Table 1. Also, note that (3.1) is similar to (1.1) with the additional condition $X_{k: n} \leq t$.

The result below shows that, as one discrete distribution, the vector of coefficients in (3.1),

$$
\boldsymbol{p}(t, k)=\left(0, \ldots, 0, p_{k+1}(t, k), \ldots, p_{n}(t, k)\right),
$$

is increasing in $t \geq 0$ in the sense of the usual stochastic order.

Theorem 3.2. Suppose that the coherent system concerned $\boldsymbol{p}(t, k)$ consists of components with i.i.d. lifetimes. Then, $\boldsymbol{p}\left(t_{1}, k\right) \leq_{\mathrm{st}} \boldsymbol{p}\left(t_{2}, k\right)$ for $t_{2}>t_{1} \geq 0$ and $k=1, \ldots, n-1$.

Proof. By definition, $\boldsymbol{p}\left(t_{1}, k\right) \leq_{\text {st }} \boldsymbol{p}\left(t_{2}, k\right)$ holds if and only if

$$
\sum_{j=s}^{n} p_{j}\left(t_{1}, k\right) \leq \sum_{j=s}^{n} p_{j}\left(t_{2}, k\right)
$$

for any $s=k+1, \ldots, n$. By virtue of (3.2), (3.3) is equivalent to

$$
\frac{\sum_{j=s}^{n} p_{j} \mathbb{P}\left(X_{j: n}>t_{1}, X_{k: n} \leq t_{1}\right)}{\sum_{i=k+1}^{n} p_{i} \mathbb{P}\left(X_{i: n}>t_{1}, X_{k: n} \leq t_{1}\right)} \leq \frac{\sum_{j=s}^{n} p_{j} \mathbb{P}\left(X_{j: n}>t_{2}, X_{k: n} \leq t_{2}\right)}{\sum_{i=k+1}^{n} p_{i} \mathbb{P}\left(X_{i: n}>t_{2}, X_{k: n} \leq t_{2}\right)} \text {. }
$$


TABLE 1: Coefficients in (3.1) for four-component systems.

\begin{tabular}{ccc}
\hline$T(\boldsymbol{X})=T\left(X_{1}, \ldots, X_{n}\right)$ & $\boldsymbol{p}=\left(p_{1}, p_{2}, p_{3}, p_{4}\right)$ & $\boldsymbol{p}(t, 1)$ \\
\hline$X_{2: 4}$ & $(0,1,0,0)$ & $(0,1,0,0)$ \\
$\min \left\{X_{1}, \max \left\{X_{2}, X_{3}, X_{4}\right\}\right\}$ & $\left(\frac{1}{4}, \frac{1}{4}, \frac{1}{2}, 0\right)$ & $\left(0, \frac{\bar{F}(t)}{3}, \frac{3-\bar{F}(t)}{3}, 0\right)$ \\
$\max \left\{\min \left\{X_{1}, X_{2}\right\}, \min \left\{X_{3}, X_{4}\right\}\right\}$ & $\left(0, \frac{2}{3}, \frac{1}{3}, 0\right)$ & $\left(0, \frac{4 \bar{F}(t)}{3+3 \bar{F}(t)}, \frac{3-\bar{F}(t)}{3+3 \bar{F}(t)}, 0\right)$ \\
$\min \left\{\max \left\{X_{1}, X_{2}\right\}, \max \left\{X_{3}, X_{4}\right\}\right\}$ & $\left(0, \frac{1}{3}, \frac{2}{3}, 0\right)$ & $\left(0, \frac{\bar{F}(t)}{3}, \frac{3-\bar{F}(t)}{3}, 0\right)$ \\
$\max \left\{X_{1}, \min \left\{X_{2}, X_{3}, X_{4}\right\}\right\}$ & $\left(0, \frac{1}{2}, \frac{1}{4}, \frac{1}{4}\right)$ & $\frac{\left(0,4 \bar{F}^{2}(t), 3 \bar{F}(t)-\bar{F}^{2}(t), 2-\bar{F}(t)+\bar{F}^{2}(t)\right)}{2+2 \bar{F}(t)+4 \bar{F}^{2}(t)}$ \\
$X_{3: 4}$ & $(0,0,1,0)$ & $(0,0,1,0)$ \\
$X_{4: 4}=\max \left\{X_{1}, X_{2}, X_{3}, X_{4}\right\}$ & $(0,0,0,1)$ & $(0,0,0,1)$ \\
\hline
\end{tabular}

Define

$$
\bar{H}_{j}(k, t)=\mathbb{P}\left(X_{j: n}>t, X_{k: n} \leq t\right) .
$$

It is sufficient to prove that

$$
\sum_{j=s}^{n} \sum_{i=k+1}^{n} p_{j} p_{i}\left[\bar{H}_{j}\left(k, t_{1}\right) \bar{H}_{i}\left(k, t_{2}\right)-\bar{H}_{j}\left(k, t_{2}\right) \bar{H}_{i}\left(k, t_{1}\right)\right] \leq 0 .
$$

Since

$$
\sum_{j=s}^{n} \sum_{i=s}^{n} p_{j} p_{i}\left[\bar{H}_{j}\left(k, t_{1}\right) \bar{H}_{i}\left(k, t_{2}\right)-\bar{H}_{j}\left(k, t_{2}\right) \bar{H}_{i}\left(k, t_{1}\right)\right]=0,
$$

we need only prove that

$$
\sum_{j=s}^{n} \sum_{i=k+1}^{s-1} p_{j} p_{i}\left[\bar{H}_{j}\left(k, t_{1}\right) \bar{H}_{i}\left(k, t_{2}\right)-\bar{H}_{j}\left(k, t_{2}\right) \bar{H}_{i}\left(k, t_{1}\right)\right] \leq 0 .
$$

Note that

$$
\bar{H}_{j}(k, t)=\mathbb{P}\left(X_{j: n}>t, X_{k: n} \leq t\right)=\sum_{m=k}^{j-1}\left(\begin{array}{l}
n \\
m
\end{array}\right) \bar{F}^{n-m}(t) F^{m}(t),
$$

and define $\phi(t)=F(t) / \bar{F}(t)$. Then inequality (3.4) can be rewritten as

$$
\sum_{j=s}^{n} \sum_{i=k+1}^{s-1} p_{j} p_{i} \sum_{m=k}^{j-1} \sum_{l=k}^{i-1}\left(\begin{array}{l}
n \\
m
\end{array}\right)\left(\begin{array}{l}
n \\
l
\end{array}\right)\left[\phi^{m}\left(t_{1}\right) \phi^{l}\left(t_{2}\right)-\phi^{m}\left(t_{2}\right) \phi^{l}\left(t_{1}\right)\right] \leq 0 .
$$

Observe that

$$
\sum_{m=k}^{i-1} \sum_{l=k}^{i-1}\left(\begin{array}{l}
n \\
m
\end{array}\right)\left(\begin{array}{l}
n \\
l
\end{array}\right)\left[\phi^{m}\left(t_{1}\right) \phi^{l}\left(t_{2}\right)-\phi^{m}\left(t_{2}\right) \phi^{l}\left(t_{1}\right)\right]=0 ;
$$

it is then equivalent to prove that

$$
\sum_{j=s}^{n} \sum_{i=k+1}^{s-1} p_{j} p_{i} \sum_{m=i}^{j-1} \sum_{l=k}^{i-1}\left(\begin{array}{l}
n \\
m
\end{array}\right)\left(\begin{array}{l}
n \\
l
\end{array}\right)\left[\phi^{m}\left(t_{1}\right) \phi^{l}\left(t_{2}\right)-\phi^{m}\left(t_{2}\right) \phi^{l}\left(t_{1}\right)\right] \leq 0 .
$$


Now, since $\phi(t)$ is an increasing function of $t$, it holds that, for all $t_{1} \leq t_{2}$ and $m>l$,

$$
\phi^{m}\left(t_{1}\right) \phi^{l}\left(t_{2}\right)-\phi^{m}\left(t_{2}\right) \phi^{l}\left(t_{1}\right) \leq 0 .
$$

This invokes the previous inequality and the desired result follows immediately.

The following result gives the tail stochastic behavior of a coherent system.

Theorem 3.3. Assume that a coherent system has signature $\boldsymbol{p}=\left(p_{1}, \ldots, p_{i}, 0, \ldots, 0\right)$ with $p_{i}>0$ for some integer $i \in\{1, \ldots, n\}$. Then

$$
\lim _{t \rightarrow \infty} p(t, k)=(\underbrace{0, \ldots, 0}_{i-1}, 1, \underbrace{0, \ldots, 0}_{n-i}) \text { for } k=1, \ldots, i-1 .
$$

Proof. From (3.2), we have

$$
p_{j}(t, k)=\frac{p_{j} \mathbb{P}\left(X_{j: n}>t, X_{k: n} \leq t\right)}{\sum_{r=k+1}^{i} p_{r} \mathbb{P}\left(X_{r: n}>t, X_{k: n} \leq t\right)} .
$$

Note that $p_{j}(t, k)=0$ for $j>i$. Then we need to only consider the $j \leq i$ case. By (3.5), it follows that

$$
\begin{aligned}
p_{j}(t, k) & =\frac{p_{j} \sum_{m=k}^{j-1}\left(\begin{array}{l}
n \\
m
\end{array}\right) \bar{F}^{n-m}(t) F^{m}(t)}{\sum_{r=k+1}^{i} p_{r} \sum_{l=k}^{r-1}\left(\begin{array}{l}
n \\
l
\end{array}\right) \bar{F}^{n-l}(t) F^{l}(t)} \\
& =\frac{p_{j} \sum_{m=k}^{j-1}\left(\begin{array}{l}
n \\
m
\end{array}\right) \phi^{m}(t)}{\sum_{r=k+1}^{i} p_{r} \sum_{l=k}^{r-1}\left(\begin{array}{c}
n \\
l
\end{array}\right) \phi^{l}(t)} \\
& =\frac{p_{j}\left(\begin{array}{l}
n \\
k
\end{array}\right) \phi^{k}(t)+p_{j}\left(\begin{array}{c}
n \\
k+1
\end{array}\right) \phi^{k+1}(t)+\cdots+p_{j}\left(\begin{array}{c}
n \\
j-1
\end{array}\right) \phi^{j-1}(t)}{\left(\sum_{r=k+1}^{i} p_{r}\right)\left(\begin{array}{l}
n \\
k
\end{array}\right) \phi^{k}(t)+\left(\sum_{r=k+2}^{i} p_{r}\right)\left(\begin{array}{c}
n \\
k+1
\end{array}\right) \phi^{k+1}(t)+\cdots+p_{i}\left(\begin{array}{c}
n \\
i-1
\end{array}\right) \phi^{i-1}(t)},
\end{aligned}
$$

where $\phi(t)=F(t) / \bar{F}(t)$. Since $\lim _{t \rightarrow \infty} \phi(t)=\infty$, then

$$
\lim _{t \rightarrow \infty} p_{j}(t, k)= \begin{cases}0, & j<i \\ 1, & j=i\end{cases}
$$

which proves the result.

The next theorem extends Theorem 2.1 of [13] to the case of the conditional residual lifetime.

Theorem 3.4. Suppose that $T_{i}(\boldsymbol{X})$ is the lifetime of a coherent system with i.i.d. components having lifetimes $X_{1}, X_{2}, \ldots, X_{n}$ and corresponding mixing coefficients vector $\boldsymbol{p}_{i}(t, k), i=$ 1,2 . Then, for any $t \geq 0, \boldsymbol{p}_{1}(t, k) \leq_{\mathrm{st}}\left(\leq_{\mathrm{hr}}, \leq_{\mathrm{lr}}\right) \boldsymbol{p}_{2}(t, k)$ implies that

$$
\left[T_{1}(\boldsymbol{X})-t \mid T_{1}(\boldsymbol{X})>t, X_{k: n} \leq t\right] \leq_{\mathrm{st}}\left(\leq_{\mathrm{hr}}, \leq_{\mathrm{lr}}\right)\left[T_{2}(\boldsymbol{X})-t \mid T_{2}(\boldsymbol{X})>t, X_{k: n} \leq t\right] .
$$

Proof. By Corollary 3.1 of [21] we have, for any $1 \leq k<j \leq n$ and $t>0$,

$$
\left[X_{j: n}-t \mid X_{j: n}>t, X_{k: n} \leq t\right] \leq \operatorname{lr}\left[X_{j+1: n}-t \mid X_{j+1: n}>t, X_{k: n} \leq t\right] .
$$

Hence,

$$
\left[X_{j: n}-t \mid X_{j: n}>t, X_{k: n} \leq t\right] \leq_{\mathrm{st}}\left(\leq_{\mathrm{hr}}\right)\left[X_{j+1: n}-t \mid X_{j+1: n}>t, X_{k: n} \leq t\right] .
$$

The proof follows from (3.1) and the mixture preservation results given in [19]. 
For two groups of i.i.d. random variables $X_{1}, X_{2}, \ldots, X_{n}$ and $Y_{1}, Y_{2}, \cdots, Y_{n}$, Zhang [21], Kochar et al. [8], and Goliforushani et al. [5] respectively showed that $X_{1} \leq_{\mathrm{hr}} Y_{1}$ implies that

$$
\left[X_{j: n}-t \mid X_{k: n} \leq t<X_{j: n}\right] \leq_{\mathrm{st}}\left[Y_{j: n}-t \mid Y_{k: n} \leq t<Y_{j: n}\right]
$$

for all $1 \leq k<j \leq n$.

Before proceeding to the second main result, we address the following two useful results, which are also of independent interest in the sense of strengthening the above usual stochastic order to the hazard rate order and likelihood ratio order, respectively.

Theorem 3.5. If $X_{1} \leq \mathrm{hr} Y_{1}$ then, for all $1 \leq k<j \leq n$,

$$
\left[X_{j: n}-t \mid X_{k: n} \leq t<X_{j: n}\right] \leq \mathrm{hr}\left[Y_{j: n}-t \mid Y_{k: n} \leq t<Y_{j: n}\right] .
$$

Proof. Note that

$$
\mathbb{P}\left(X_{j: n}-t>x \mid X_{1: n}>t\right)=\int_{F_{t}(x)}^{1} \frac{n !}{(j-1)(n-j) !} u^{j-1}(1-u)^{n-j} \mathrm{~d} u
$$

(see [5]) for all $j \geq 1$, where $F_{t}(x)=1-\bar{F}(t+x) / \bar{F}(t)$.

Define $\phi_{1}(t)=F(t) / \bar{F}(t)$ and $\phi_{2}(t)=G(t) / \bar{G}(t)$. Then

$$
\begin{aligned}
\mathbb{P}\left(X_{j: n}-t>x \mid X_{k: n} \leq t<X_{j: n}\right) & \\
& =\frac{\sum_{l=k}^{j-1} \mathbb{P}\left(X_{j: n}>t+x \mid X_{l: n}<t<X_{l+1: n}\right) \mathbb{P}\left(X_{l: n}<t<X_{l+1: n}\right)}{\mathbb{P}\left(X_{k: n} \leq t<X_{j: n}\right)} \\
& =\frac{\sum_{l=k}^{j-1} \mathbb{P}\left(X_{j-l: n-l}>t+x \mid X_{1: n-l}>t\right)\left(\begin{array}{l}
n \\
l
\end{array}\right) \bar{F}^{n-l}(t) F^{l}(t)}{\sum_{m=k}^{j-1}\left(\begin{array}{l}
n \\
m
\end{array}\right) \bar{F}^{n-m}(t) F^{m}(t)} \\
& =\frac{\sum_{l=k}^{j-1} \mathbb{P}\left(X_{j-l: n-l}>t+x \mid X_{1: n-l}>t\right)\left(\begin{array}{l}
n \\
l
\end{array}\right) \phi_{1}^{l}(t)}{\sum_{m=k}^{j-1}\left(\begin{array}{l}
n \\
m
\end{array}\right) \phi_{1}^{m}(t)} \\
& =\frac{\int_{0}^{1} \mathbf{1}\left(F_{t}(x) \leq u \leq 1\right) \sum_{l=k}^{j-1}\left(\begin{array}{l}
n \\
l
\end{array}\right)\left(\begin{array}{l}
n-l \\
j-l
\end{array}\right)(j-l) \phi_{1}^{l}(t) u^{j-l-1}(1-u)^{n-j} \mathrm{~d} u}{\sum_{m=k}^{j-1}\left(\begin{array}{l}
n \\
m
\end{array}\right) \phi_{1}^{m}(t)} .
\end{aligned}
$$

Likewise, $\mathbb{P}\left(Y_{j: n}-t>x \mid Y_{k: n} \leq t<Y_{j: n}\right)$ may be represented in a similar manner. Note that

$$
\begin{aligned}
& \frac{\mathbb{P}\left(Y_{j: n}-t>x \mid Y_{k: n} \leq t<Y_{j: n}\right)}{\mathbb{P}\left(X_{j: n}-t>x \mid X_{k: n} \leq t<X_{j: n}\right)} \\
& \quad \propto \frac{\int_{0}^{1} \mathbf{1}\left(G_{t}(x) \leq u \leq 1\right) \sum_{l=k}^{j-1}\left(\begin{array}{c}
n \\
l
\end{array}\right)\left(\begin{array}{c}
n-l \\
j-l
\end{array}\right)(j-l) \phi_{2}^{l}(t) u^{j-l-1}(1-u)^{n-j} \mathrm{~d} u}{\int_{0}^{1} \mathbf{1}\left(F_{t}(x) \leq u \leq 1\right) \sum_{l=k}^{j-1}\left(\begin{array}{c}
n \\
l
\end{array}\right)\left(\begin{array}{c}
n-l \\
j-l
\end{array}\right)(j-l) \phi_{1}^{l}(t) u^{j-l-1}(1-u)^{n-j} \mathrm{~d} u} \\
& \quad \propto \mathbb{E}_{x}[\psi(U, x)],
\end{aligned}
$$

where, for $F_{t}(x) \leq u<1$,

$$
\psi(u, x)=\frac{\mathbf{1}\left(G_{t}(x) \leq u \leq 1\right) \sum_{l=k}^{j-1}\left(\begin{array}{c}
n \\
l
\end{array}\right)\left(\begin{array}{c}
n-l \\
j-l
\end{array}\right)(j-l) \phi_{2}^{l}(t) u^{j-l-1}}{\mathbf{1}\left(F_{t}(x) \leq u \leq 1\right) \sum_{l=k}^{j-1}\left(\begin{array}{c}
n \\
l
\end{array}\right)\left(\begin{array}{c}
n-l \\
j-l
\end{array}\right)(j-l) \phi_{1}^{l}(t) u^{j-l-1}}
$$


is increasing in both $x$ and $u$ by $X \leq_{\mathrm{hr}} Y$ and Lemma 2.3, and the distribution function of the nonnegative random variable $U$ belongs to the family $\mathscr{H}=\{H(\cdot \mid x), x \in \mathbb{X}\}$ with densities

$$
h(u \mid x)=c(x) \mathbf{1}\left(F_{t}(x) \leq u \leq 1\right) \sum_{l=k}^{j-1}\left(\begin{array}{l}
n \\
l
\end{array}\right)\left(\begin{array}{c}
n-l \\
j-l
\end{array}\right)(j-l) \phi_{1}^{l}(t)(1-u)^{n-j} u^{j-l-1}
$$

with some normalizing constant $c(x)$. Since $h(u \mid x)$ is $\mathrm{TP}_{2}$ in $(u, x) \in \mathbb{R}_{+}^{2}$, this implies that $H\left(\cdot \mid x_{2}\right) \geq \operatorname{lr} H\left(\cdot \mid x_{1}\right)$ and, hence,

$$
H\left(\cdot \mid x_{2}\right) \geq_{\text {st }} H\left(\cdot \mid x_{1}\right) \quad \text { for } x_{2} \geq x_{1}>0 .
$$

From Lemma 2.1, it follows that

$$
\mathbb{E}_{x_{1}} \psi\left(U, x_{1}\right) \leq \mathbb{E}_{x_{2}} \psi\left(U, x_{2}\right) \text { for } x_{2} \geq x_{1}>0 .
$$

Thus,

$$
\frac{\mathbb{P}\left(Y_{j: n}-t>x \mid Y_{k: n} \leq t<Y_{j: n}\right)}{\mathbb{P}\left(X_{j: n}-t>x \mid X_{k: n} \leq t<X_{j: n}\right)}
$$

is increasing in $x$ for any $t \geq 0$. This completes the proof.

The next conclusion can be directly obtained from Theorem 3.7 of [1] or Theorem 3.2 of [2].

Theorem 3.6. If $X_{1} \leq \operatorname{lr} Y_{1}$ then, for all $1 \leq k<j \leq n$,

$$
\left[X_{j: n}-t \mid X_{k: n} \leq t<X_{j: n}\right] \leq \operatorname{lr}\left[Y_{j: n}-t \mid Y_{k: n} \leq t<Y_{j: n}\right] .
$$

As the second main result, Theorem 3.7 below provides sufficient conditions for the conditional residual lifetime of one coherent system to be larger than that of another coherent system in three different senses: the usual stochastic order, hazard rate order, and likelihood ratio order.

Theorem 3.7. Suppose that $\boldsymbol{X}=\left(X_{1}, \ldots, X_{n}\right)$ and $\boldsymbol{Y}=\left(Y_{1}, \ldots, Y_{n}\right)$ are lifetimes of two groups of i.i.d. components, and coherent systems $T_{1}(\boldsymbol{X})$ and $T_{2}(\boldsymbol{Y})$ have their corresponding mixing coefficients vector $\boldsymbol{p}_{i}(t, k)=\left(0, \ldots, 0, p_{i, k+1}(t, k), \ldots, p_{i, n}(t, k)\right), i=1,2$. Then, for any $t \geq 0$,

(i) $X_{1} \leq_{\mathrm{hr}} Y_{1}$ and $\boldsymbol{p}_{1}(t, k) \leq_{\mathrm{st}}\left(\leq_{\mathrm{hr}}\right) \boldsymbol{p}_{2}(t, k)$ imply that

$$
\left[T_{1}(\boldsymbol{X})-t \mid T_{1}(\boldsymbol{X})>t, X_{k: n} \leq t\right] \leq_{\mathrm{st}}\left(\leq_{\mathrm{hr}}\right)\left[T_{2}(\boldsymbol{Y})-t \mid T_{2}(\boldsymbol{Y})>t, Y_{k: n} \leq t\right] ;
$$

(ii) $X_{1} \leq_{\operatorname{lr}} Y_{1}$ and $\boldsymbol{p}_{1}(t, k) \leq \operatorname{lr} \boldsymbol{p}_{2}(t, k)$ imply that

$$
\left[T_{1}(\boldsymbol{X})-t \mid T_{1}(\boldsymbol{X})>t, X_{k: n} \leq t\right] \leq_{\operatorname{lr}}\left[T_{2}(\boldsymbol{Y})-t \mid T_{2}(\boldsymbol{Y})>t, Y_{k: n} \leq t\right] .
$$

Proof. By the mixture representation (3.1),

$$
\begin{aligned}
& \mathbb{P}\left(T_{1}(\boldsymbol{X})-t>x \mid T_{1}(\boldsymbol{X})>t, X_{k: n} \leq t\right) \\
& \quad=\sum_{j=k+1}^{n} p_{1, j}(t, k) \mathbb{P}\left(X_{j: n}-t>x \mid X_{j: n}>t, X_{k: n} \leq t\right), \\
& \mathbb{P}\left(T_{2}(\boldsymbol{Y})-t>x \mid T_{2}(\boldsymbol{Y})>t, Y_{k: n} \leq t\right) \\
& \quad=\sum_{j=k+1}^{n} p_{2, j}(t, k) \mathbb{P}\left(Y_{j: n}-t>x \mid Y_{j: n}>t, Y_{k: n} \leq t\right) .
\end{aligned}
$$


According to Theorem 3.5, $X_{1} \leq_{\mathrm{hr}} Y_{1}$ implies that, for all $1 \leq k<j \leq n$,

$$
\left[X_{j: n}-t \mid X_{k: n} \leq t<X_{j: n}\right] \leq_{\mathrm{hr}}\left[Y_{j: n}-t \mid Y_{k: n} \leq t<Y_{j: n}\right],
$$

and, hence,

$$
\left[X_{j: n}-t \mid X_{k: n} \leq t<X_{j: n}\right] \leq_{\mathrm{st}}\left[Y_{j: n}-t \mid Y_{k: n} \leq t<Y_{j: n}\right] .
$$

As a consequence, the desired stochastic order and the hazard rate order follow from Theorems 1.A.6 and 1.B.14 of [19], respectively.

From Theorem 3.6, $X_{1} \leq_{\text {rr }} Y_{1}$ implies that

$$
\left[X_{j: n}-t \mid X_{k: n} \leq t<X_{j: n}\right] \leq \operatorname{lr}\left[Y_{j: n}-t \mid Y_{k: n} \leq t<Y_{j: n}\right]
$$

for all $1 \leq k<j \leq n$. Then, the likelihood ratio order directly follows from Theorem 1.C.17 of [19].

To close, we present a numerical example which indicates that the order between $\boldsymbol{p}_{1}(t, k)$ and $\boldsymbol{p}_{2}(t, k)$ in Theorem 3.7 is necessary.

Example 3.1. Suppose that $X_{1}, X_{2}, X_{3}$ and $Y_{1}, Y_{2}, Y_{3}$ are two sets of i.i.d. copies of $X$ and $Y$, respectively. Let $X$ and $Y$ have the following respective density functions:

$$
f(x)=1.2 \mathrm{e}^{-1.2 x}, \quad g(x)=\mathrm{e}^{-x},
$$

for $x \geq 0$. The systems $T_{1}(\boldsymbol{X})=\max \left\{\min \left\{X_{1}, X_{2}\right\}, X_{3}\right\}$ and $T_{2}(\boldsymbol{Y})=\max \left\{\min \left\{Y_{1}, Y_{2}\right\}, Y_{3}\right\}$ have the same signature $\boldsymbol{p}=\left(0, \frac{2}{3}, \frac{1}{3}\right)$ and the corresponding mixing coefficients vectors are

$$
p_{1}(t, 1)=\left(0, \frac{2 \bar{F}(t)}{2 \bar{F}(t)+1}, \frac{1}{2 \bar{F}(t)+1}\right)
$$

and

$$
p_{2}(t, 1)=\left(0, \frac{2 \bar{G}(t)}{2 \bar{G}(t)+1}, \frac{1}{2 \bar{G}(t)+1}\right) .
$$

Let $f_{1,3, t}(x)$ and $g_{1,3, t}(x)$ be the density functions of the random variables $\left[T_{1}(\boldsymbol{X})-t \mid T_{1}(\boldsymbol{X})>\right.$ $\left.t, X_{1: 3} \leq t\right]$ and $\left[T_{2}(\boldsymbol{Y})-t \mid T_{2}(\boldsymbol{Y})>t, Y_{1: 3} \leq t\right]$, respectively. By some computations we have

$$
\frac{g_{1,3, t}(x)}{f_{1,3, t}(x)} \propto \frac{\mathrm{e}^{-(t+x)}\left(1+2 \mathrm{e}^{-(t+x)}+\mathrm{e}^{-t}\right)}{\mathrm{e}^{-1.2(t+x)}\left(1+2 \mathrm{e}^{-1.2(t+x)}+\mathrm{e}^{-1.2 t}\right)}=\Delta(t, x) .
$$

Note that $X \leq \operatorname{lr} Y$, but $\boldsymbol{p}_{1}(t, 1) \not \operatorname{lr} \boldsymbol{p}_{2}(t, 1)$, and, for $t=1$,

$$
\Delta(1,1)=3.62418>3.53823=\Delta(1,1.5),
$$

that is, $\left[T_{1}(\boldsymbol{X})-t \mid T_{1}(\boldsymbol{X})>t, X_{1: 3} \leq t\right] \not \operatorname{lr}_{\mathrm{r}}\left[T_{2}(\boldsymbol{Y})-t \mid T_{2}(\boldsymbol{Y})>t, Y_{1: 3} \leq t\right]$.

\section{Acknowledgements}

We are thankful to an anonymous referee and the editor for their careful reading and thoughtful comments on a previous version of this paper. Dr Zhang's research was financially supported by the National Natural Science Foundation of China (grant number TY11126182), and Dr Li's research was financially supported by the National Natural Science Foundation of China (grant number 11171278) and the Fundamental Research Funds for the Central Universities (lzujbky-2013-13). 


\section{References}

[1] Balakrishnan, N., Belzunce, F., Hami, N. and Khaledi, B. E. (2010). Univariate and multivariate likelihood ratio ordering of generalized order statistics and associated conditional variables. Prob. Eng. Inf. Sci. 24, 441455.

[2] Belzunce, F., Gurler, S. And Ruiz, J. M. (2011). Revisiting multivariate likelihood ratio ordering results for order statistics. Prob. Eng. Inf. Sci. 25, 355-368.

[3] Eryilmaz, S. (2013). On residual lifetime of coherent systems after the $r$ th failure. Statist. Papers 54, $243-250$.

[4] Gertsbakh, I., Shpungin, Y. and Spizzichino, F. (2012). Two-dimensional signatures. J. Appl. Prob. 49, 416-429.

[5] Goliforushani, S., Asadi, M. and Balakrishnan, N. (2012). On the residual and inactivity times of the components of used coherent systems. J. Appl. Prob. 49, 385-404.

[6] Karlin, S. (1968). Total Positivity, Vol. I. Stanford University Press.

[7] Khaledi, B.-E. And ShaKed, M. (2007). Ordering conditional lifetimes of coherent systems. J. Statist. Planning Infer. 137, 1173-1184.

[8] Kochar, S. ANd Xu, M. (2010). On residual lifetimes of $k$-out-of- $n$ systems with nonidentical components. Prob. Eng. Inf. Sci. 24, 109-127.

[9] Kochar, S., Mukerjee, H. and Samaniego, F. J. (1999). The "signature" of a coherent system and its application to comparisons among systems. Naval Res. Logistics 46, 507-523.

[10] Li, X. And Zhang, Z. (2008). Some stochastic comparisons of conditional coherent systems. Appl. Stoch. Models Business Industry 24, 541-549.

[11] Mahmoudi, M. And Asadi, M. (2011). The dynamic signature of coherent systems. IEEE Trans. Reliab. 60, 817-822.

[12] Misra, N. and van der Meulen, E. C. (2003). On stochastic properties of $m$-spacings. J. Statist. Planning Infer. 115, 683-697.

[13] Navarro, J., Balakrishnan, N. And Samaniego, F. J. (2008). Mixture representations of residual lifetimes of used systems. J. Appl. Prob. 45, 1097-1112.

[14] Navarro, J., Samaniego, F. J. and Balakrishnan, N. (2010). The joint signature of coherent systems with shared components. J. Appl. Prob. 47, 235-253.

[15] Navarro, J., Samaniego, F. J. and Balakrishnan, N. (2011). Signature-based representations for the reliability of systems with heterogeneous components. J. Appl. Prob. 48, 856-867.

[16] Samaniego, F. J. (1985). On closure of the IFR class under formation of coherent systems. IEEE Trans. Reliab. R-34, 69-72.

[17] Samaniego, F. J. (2007). System Signatures and Their Applications in Engineering Reliability. Springer, New York.

[18] Samaniego, F. J., Balakrishnan, N. and Navarro, J. (2009). Dynamic signatures and their use in comparing the reliability of new and used systems. Naval Res. Logistics 56, 577-591.

[19] Shaked, M. and Shanthikumar, J. G. (2007). Stochastic Orders. Springer, New York.

[20] Zhang, Z. (2010). Mixture representations of inactivity times of conditional coherent systems and their applications. J. Appl. Prob. 47, 876-885.

[21] Zhang, Z. (2011). Ordering new conditional residual lifetimes of $k$-out-of- $n$ systems. Commun. Statist. Theory Meth. 40, 1591-1600.

[22] Zhang, Z. AND LI, X. (2010). Some new result on stochastic orders and aging properties of coherent systems. IEEE Trans. Reliab. 59, 718-724. 